\title{
Trauma and the pyoderma gangrenosum of inflammatory bowel disease
}

\author{
S I FINKEL* AND H D JANOWITZ
}

From the Division of Gastroenterology of the Department of Medicine, Mount Sinai School of Medicine of the City University of New York, New York, USA

SUMMARY In five patients with inflammatory bowel disease (three with ulcerative colitis, two with Crohn's disease), pyoderma gangrenosum developed on a lower extremity at the site of trauma. In these subjects, the pyoderma was not clearly correlated with disease activity.

Pyoderma gangrenosum was considered to be a 'colitis related' extraintestinal complication of inflammatory bowel disease, occurring in $5 \%$ of patients with ulcerative colitis and in $1.4 \%$ with Crohn's colitis and ileocolitis in our recent series of 700 patients with inflammatory bowel disease. ${ }^{1}$

In general this serious skin complication is usually believed to be related to the clinical activity of the underlying bowel disease. Indeed, in a leading textbook of gastroenterology, the statement is made that 'almost invariably, these lesions appear during a bout of active colitis; healing of skin lesions will usually follow control of the colitis with either corticosteroid therapy or colectomy'. ${ }^{2}$ However, a recent report $^{3}$ makes the observation that, in six patients with ulcerative colitis and pyoderma gangrenosum, there was no correlation between exacerbation of the colitis and the onset or course of the skin disorder.

We have recently seen five patients with inflammatory bowel disease and pyoderma gangrenosum in whom antecedent trauma was a common precipitating factor and in four of whom there was no association with clinical exacerbation of disease activity.

\section{Case reports}

CASE 1

A 51 year old woman with a long history_of ulcera-

*Address for reprint requests: S I Finkel, MD, Mount Sinai School of Medicine, Annenberg 23-08, 1 Gustave L Levy Place, New York, New York 10029, USA.

Received for publications 28 November 1980 tive colitis and associated arthritis was maintained on prednisone and azathioprine. She fell and struck her right shin at a time when her disease was stable and manifested by only two to three loose stools per day, without blood or mucus, and she subsequently developed pyoderma gangrenosum at the site of trauma. The pyoderma responded to parenteral hydrocortisone and local measures.

Two years later, she fell again, striking the left thenar eminence, which developed characteristic lesions of pyoderma gangrenosum within one week. Colitic activity had been clinically quiescent before trauma and remained stable subsequently, with two to three loose, heme-negative stools per day. The pyoderma again responded to parenteral corticosteroids.

\section{CASE 2}

A 44 year old woman with a 16 year history of ulcerative colitis was maintained on prednisone. She had had previous episodes of erythema nodosum associated with exacerbations of disease activity. Pyoderma gangrenosum developed at the site of a skin laceration soon after the patient struck her left shin on a bed frame, at a time when her colitis was symptomatically quiescent. The pyoderma responded to increased doses of prednisone and parenteral antibiotics. However, her colitis flared subsequently, requiring total proctocolectomy. During this time, she also received parenteral hydrocortisone and the pyoderma continued to resolve.

\section{CASE 3}

A 24 year old woman with a six year history of ulcerative colitis had one previous episode of pyo- 
derma gangrenosum reportedly associated with antecedent trauma without exacerbation of her inflammatory bowel disease. The present episode began at a time when bowel symptoms were minimal on prednisone and salicylazosulphapyridine, and was precipitated by a fall in which the patient twisted her right ankle and struck her right shin. Pyoderma gangrenosum developed at the latter site, did not respond to parenteral hydrocortisone, antibiotics, or local measures, and prompted total proctocolectomy. The pyoderma did not respond to surgery but extended as steroids were tapered. After a prolonged stay in hospital, the pyoderma responed slowly to high dose prednisone, antibiotics, and zinc sulphate, as blood zinc levels were noted to be low.

\section{CASE 4}

A 20 year old woman with a two year history of granulomatous ileocolitis was asymptomatic while being maintained on salicylazosulphapyridine

Pyoderma gangrenosum developed over the left medial malleolus after she twisted this ankle. The lesion responded to parenteral hydrocortisone and local measures.

\section{CASE 5}

A 46 year old man had a 30 year history of granulomatous ileocolitis requiring multiple ileal resections and a right hemicolectomy in the past. For two years before admission, he had frequent recurrences of bloody diarrhoea requiring multiple transfusions and maintenance on prednisone. Six months before admission, he struck his right shin on the fender of his car and developed pyoderma gangrenosum at that site. He was admitted in haemorrhagic shock and coma and required transfusions, parenteral hydrocortisone, and antibiotics. His pyoderma was of minimal extent in spite of fulminant inflammatory bowel disease. His course was complicated by respiratory failure requiring ventilatory support, and recurrent rectal haemorrhaging prompting emergency transverse colectomy and partial ileal resection. The patient died postoperatively after his illness had run a septic course with recurrent grand mal seizures.

\section{Discussion}

Colitic activity as judged by symptoms was not increased at the time of onset of pyoderma gangrenosum in four or five patients, although, in case 2, colitic activity subsequently flared as the pyoderma responded to therapy. It is possible that, in this patient, the appearance of pyoderma gangrenosum was a harbinger of exacerbation of her inflammatory bowel disease; some previous exacerbations had been heralded by erythema nodosum. However, trauma was directly related to the onset of pyoderma in this subject, but it should not be inferred that the subsequent exacerbation was a consequence of the traumatic episode. Only case 5 developed pyoderma gangrenosum in association with a long period of recurrent exacerbations, but, again, the pyoderma was secondary to trauma.

In these five patients, antecedent trauma occurred at the site on a lower extremity where pyoderma gangrenosum subsequently developed; in one, it also developed on the hand, also at the site of injury. If circulating immune complexes, which have been described in patients with inflammatory bowel disease, ${ }^{45}$ play a role in the development of extraintestinal manifestations of inflammatory bowel disease, then trauma-induced vascular damage might contribute to localisation of the pyoderma. In this respect, it is interesting to note that four of these five patients were taking prednisone at the time of their traumatic episodes, as glucocorticoids have been reported to increase capillary resistance independent of the presence of thrombocytopenia ${ }^{6}$ ? and therefore might have been expected to exert some protective effect. Such an effect could possibly be antagonised by glucocorticoid interference with wound healing, permitting development of the chronic ulcer characteristic of pyoderma gangrenosum at sites of trauma.

Pyoderma gangrenosum generally responds to treatment of the underlying inflammatory bowel disease. Only one of our patients, case 3, failed to demonstrate improvement of her pyoderma with either parenteral steroids or colectomy. Partial response was obtained only after prolonged hospitalisation and several different methods of treatment. Case 5, who died postoperatively of the complications of fulminant inflammatory bowel disease, had minimal pyoderma gangrenosum which was not a significant clinical problem.

The additional observation of a variable relationship between erythema nodosum and activity of Crohn's colitis and ileocolitis ${ }^{8}$ is of interest in light of Thornton et al. $^{3}$ and our failure to confirm a clear association between pyoderma gangrenosum and inflammatory bowel disease. Jacobs and his co-authors ${ }^{8}$ noted that five of 12 patients with Crohn's disease and erythema nodosum developed no change in clinical activity of their inflammatory bowel disease within one month of onset of the skin manifestation, and concluded that erythema nodosum does not always indicate that other manifestations of Crohn's disease will worsen.

While pyoderma gangrenosum in our experience is usually a 'colitis-related' complication of inflam- 
matory bowel disease, if it cannot be related to clinical exacerbations of disease trauma should be sought as a possible precipitating factor.

\section{References}

${ }^{1}$ Greenstein AJ, Janowitz HD, Sachar DB. The extraintestinal complications of Crohn's disease and ulcerative colitis: a study of 700 patients. Medicine 1976; 55: 401-12. ${ }^{2}$ Meye JH. Ulcerative colitis. In: Sleisinger MH, Fordtran JS, eds. Gastrointestinal disease. Philadelphia, 1973: 1379.

${ }^{3}$ Thornton JR, Teague RH, Lon-Beer TS, Read AE. Pyoderma gangrenosum and ulcerative colitis. Gut 1979; 21 : $247-8$.
${ }^{4} J$ Jewell DP, Mac Lennan ICM. Circulating immune complexes in inflammatory bowel disease. Clin Exp Immunol 1973; 14: 219-26.

${ }^{5}$ Kemler BJ, Alpert E. Inflammatory bowel disease: Essential circulating immune complexes. Gut 1980; 21: 195-201.

${ }^{6}$ Robson HN, Duthie JJR. Capillary resistance and adrenocortical activity. $\mathrm{Br}$ Med $J$ 1950; 2: 971-7.

${ }^{7}$ Faloon WW, Green RW, Lozner EL. The hemostatic defect in thrombocytopenia as studied by the use of ACTH and cortisone. Am J Med 1952; 13: 12-9.

${ }^{8}$ Jacobs M, Winkelman EL, Farmer RG. Relationship of erythema nodosum to other manifestations of Crohn's disease. Cleve Clin $Q$ 1977; 44: 145-8. 